\title{
EMDR - Entwicklung, Praxis und Veränderung
}

\author{
Eva Münker-Kramer (D)
}

Online publiziert: 8. Juni 2017

(c) Der/die Autor(en) 2017. Dieser Artikel ist eine Open-Access-Publikation.

Zusammenfassung Eine Besonderheit bei EMDR der vor fast 30 Jahren von Dr. Francine Shapiro beschriebenen Methode zur Behandlung psychischer Traumatisierungen - ist, dass sie abgesehen von wenigen grundsätzlichen Paradigmenwechseln kaum Abweichungen zwischen Theorie und Praxis vorsieht. Dies ist ein Grundaspekt und Grundpfeiler ihrer Wirksamkeit und Programmatik.

Ein Kurzeinblick in die Methode an sich sowie die o.g. Besonderheit und dessen Hintergrund sollen in diesem Artikel beschrieben werden. So werden die Charakteristika von EMDR in einem Abriss erläutert und daraus einerseits starke Standardisierung und andererseits Überlegungen zu Modifikationen dargestellt.

Schlüsselwörter EMDR - Traumatherapie - PTBS · Pathogene Erinnerungen · AIP-Modell

\section{EMDR - development, practical application and change}

Summary One of the most determining factors of EMDR - that was brought up by Francine Shapiro, PhD nearly 30 years ago - is that theory and practice have ever been very much alike. This is - besides some major changes in its development - crucial for its effectiveness. EMDR itself and its background will briefly be illustrated in this article. Thus the characteristics will be outlined to prepare the explanation of high standardization and reflections on modifications.

Diese Arbeit ist Teil des Leitthemas „Traumatherapie in der Praxis“.

\section{E. Münker-Kramer $(\bowtie)$}

Klinisch-psychologisch/psychotherapeutische

Praxis und EMDR Institut Austria,

Brandströmstr. 7/7, 3500 Krems/Donau, Österreich

muenker-kramer@emdr-institut.at
Keywords EMDR · Traumatherapy · PTSD - Dysfunctional memories · AIP model

\section{EMDR als Psychotherapie zur Bearbeitung traumatischer Erfahrungen}

Um was geht es?

EMDR (Eye Movement Desensitization and Reprocessing) ist in Europa in zwei Ländern (Großbritannien, Deutschland) durch staatliche Gesundheitsrichtlinien (UK: NICE Report 2005; Bisson 2013; D: Schulz et al. 2015) als Richtlinienpsychotherapie für PTBS (Posttraumatische Belastungsstörung) anerkannt (G-BA 2017).

Die Entwicklung von EMDR geht auf die Arbeiten der kalifornischen Psychologin und Verhaltenstherapeutin Dr. Francine Shapiro Ende der 1980er-Jahre zurück. Sie beschrieb eine Möglichkeit, belastende Erinnerungen mittels einerseits bilateraler Stimulation der Augen und andererseits Fokussierung auf die vier wesentlichen Repräsentationsebenen - Bild, Kognition, Emotion und Körperkorrelat - zu entlasten.

Da diese Überlegungen und die ersten Publikationen dazu nachvollziehbarerweise sehr skeptisch betrachtet wurden, initiierte und führte sie selbst seitdem sehr viele kontrollierte Studien zur Wirksamkeitsforschung und $\mathrm{zu}$ den zugrundeliegenden Wirkmechanismen durch. Diese Entwicklung kann im Detail nachgelesen werden (z. B. Shapiro 2013; Hofmann 2014; Münker-Kramer 2015).

1995 beschrieb Shapiro das Konzept der acht Phasen des EMDR. Diese Vorgehensweise wird bis heute so aufrecht erhalten. Sie integriert die klassischen vier Phasen der Traumatherapie. Diese wurden bereits von Pierre Janet bahnbrechend und nach wie vor unbestritten aktuell (Reddeman 2005; Huber 2009; Rost 2016) im ausgehenden 19. Jahrhundert im Zu- 
ge seiner Arbeit mit Charcot in Paris (Münker-Kramer 2015) konstatiert: 1. Anamnese und Beziehungsaufbau, 2. Stabilisierung und Behandlungsplanung, 3. Traumakonfrontation (auch-Integration, Synthese u. a.) und 4. Integration und Neuorientierung.

Beim EMDR werden nach sorgfältiger Phase eins und zwei zusätzliche Zwischenstufen berücksichtigt: So wird gemeinsam mit den PatientInnen nach der Definition des $\mathrm{zu}$ bearbeitenden Problembereichs eine repräsentative Erinnerung definiert. Diese wird bezüglich der in ihr repräsentierten äußeren Belastung, der Auswirkung auf Gedanken und Einstellungen, der gefühlsmäßigen sowie der körperlichen Belastung besprochen und bewertet (Phase 3). Daraufhin wird in einem relativ freien Assoziationsprozess das sogenannte Desensibilisieren und Reprozessieren dieses Ereignisses (Phase 4) durchgeführt. Es mündet im Verankern des veränderten Erlebens, repräsentiert durch die sogenannte positive Kognition (Phase 5) und wird abgeschlossen mit der Durchführung eines Körpertests zur Absicherung, ob sich die Traumasynthese auch bis in die Repräsentationsebene Körper auswirkt (auch Traumametabolisierung genannt) (Phase 6). Eine gezielte Nachbesprechung und Psychoedukation (Phase 7) beendet die Traumakonfrontationssitzung. Unter Einbeziehung der Erfahrungen und des Erlebens des Alltags nach der Sitzung erfolgt eine Neubewertung, Neuplanung, Nachbesprechung in der Phase 8 beim nächsten Kontakt.

Letztlich geht es darum, die im Zuge des traumatischen Stresses dysfunktional gespeicherten Erinnerungen - wie z. B. im Moment des (damaligen) Erlebens „steckengebliebene“ Emotionen oder Körpersensationen - aus dieser Verbindung herauszulösen und somit als Ereignis der Vergangenheit mit seinen Begleiterscheinungen kortikal einzuordnen. Dies ist letztlich auch in anderen traumatherapeutischen Ansätzen das Ziel, rein sprachlich-logische Therapieansätze sind aber oft von limitierter Wirksamkeit. Die Wirkung von EMDR bei anderen Arten sogenannter pathogener Erinnerungen wird intensiv in den letzten Jahren beforscht (Hofmann 2014). Hier liegt ein großes Potential, und die Methode erfreut sich großen Interesses von Institutionen und in freier Praxis tätigen KlinikerInnen sowie wissenschaftlich tätiger Fachleute.

Das oben kurz beschriebene und für EMDR charakteristische Detailvorgehen über die acht Phasen ist eingebettet in eine Gesamttherapie, bei der EMDR je nach Problematik und Ausmaß der Beschwerden einen definierten Anteil hat. So kann es bei umgrenzten belastenden Erfahrungen (sogenannten Typ I Traumatisierungen) (Münker-Kramer 2009) durchaus sein, dass wenige Konfrontationssitzungen mit kurzer Vorbereitung und Nachbesprechung schon die gewünschte Entlastung bringen. Bei komplexeren Belastungsnetzwerken im Gehirn kann es wesentlich länger dauern und EMDR in verschiedenen Teiltechniken und zu verschiedenen Teilaspekten (in der Behandlungsplanung auch als Cluster bezeich- net) verwendet werden - verantwortungsbewusst und gezielt eingebettet in eine längerdauernde Psychotherapie. EMDR stellt kein psychotherapeutisches Erstverfahren dar, sondern kann lt. Richtlinien der EMDR Fachverbände als Spezifizierung seriöserweise nur von ausgebildeten KlinikerInnen erlernt werden.

\section{Wie wirkt EMDR und warum?}

Nach dieser kurzen plakativen Beschreibung des grundsätzlichen Vorgehens sei die Frage nach der klinischen Wirksamkeit und den konkreten Wirkmechanismen gestellt: Heute ist die Wirksamkeit an sich völlig unbestritten (z. B. van Etten und Taylor 1998; Power et al. 2002; Schulz et al. 2015). Dem wird Rechnung getragen in den gesundheitspolitischen Anerkennungen durch Gesundheitsbehörden und der inhaltlichen Anerkennung durch Fachgesellschaften (z. B. APA, ISTSS, WHO, Psychotherapiebeirat Deutschland, NICE, AWMF Leitlinien) seit den 1990er Jahren. Nichtsdestotrotz gibt es kritische Diskussionen um die Methode vor allem in Richtungen.

Einerseits wird angemerkt, dass die Gefahr besteht, bei unsachgemäßer Anwendung negative Effekte wie Überflutungszustände zu haben. Diese Befürchtung ist durchaus berechtigt. Man kann nur qualifizierte Ausbildung und Einbettung in Gesamttraumatherapieausbildungen anregen wie dies z. B. in den Richtlininen der Deutschspachigen Gesellschaft für Psychotraumtologie gegeben ist (www.degpt.de). Hier ist EMDR neben traumafokussierter Verhaltenstherapie zwar unter die sogenannten Verfahren mit Evidenzgrad la gereiht, wird aber im Kontext weiterer psychotraumatologischer Inhalte vermittelt. Hiermit soll sichergestellt werden, dass es nicht isoliert ohne $\mathrm{Zu}$ satzkenntnisse als Allheilmittel-Technik angewendet wird, was aber nur begrenzt möglich ist, da es weder gesetzlichen „Titelschutz“ noch „Tätigkeitsschutz“ gibt. Auch wenn der Fokus dieses Artikels bei EMDR liegt, sollen bisher nicht systematisch durch Kontrollgruppendesigns beforschte Methoden zur Traumakonfrontation an dieser Stelle nicht unerwähnt bleiben. Hier sind vor allem die Screen Technik (z. B. nach Lutz Besser und auch Michaela Huber), die Beobachtertechnik (z. B. nach Ulrich Sachsse) oder bei komplex Traumatisierten die sehr gut etablierte Psychodynamisch-Imaginative Traumatherapie (PITT) nach Reddemann zu erwähnen. Sie sind praktisch und klinisch in breiter Verwendung und aus der Gruppe klinisch weit verbreiteter Traumabehandlungsverfahren nicht wegzudenken. Es gibt in den einzelnen Fällen viele Differentialindikationen - auch für die Kombination der erwähnten Anwendungen.

Sehr stark aber auch durch die lange bestehende Konkurrenz zwischen TF-KVT (traumafokussierte kognitive Verhaltenstherapie) und EMDR sind eben diese beiden Methoden in einen Wettlauf vergleichender RCTs (Randomised controlled study - vergleichende statistische Wirksamkeitsforschung im 
Kontrollgruppendesign) eingestiegen und haben daher sowohl kontrollierte Einzelstudien als auch Metaanalysen angeregt. Demzufolge gehören beide $\mathrm{zu}$ den effektivsten und effizientesten Methoden (siehe o.g. gesundheitspolitische Richtlinien), wie es auch die erwähnte Empfehlung der WHO von 2013 illustriert: „... In addition, referral for advanced treatments such as cognitive-behavioral therapy (CBT) or a new technique called eye movement desensitization and reprocessing (EMDR) should be considered for people suffering from PTSD ..." (WHO mhGAP Guidelines 2013).

Davon abgesehen ist der zweite Kritikbereich die Tatsache, dass nach wie vor die Wirkmechanismen beim EMDR nicht klar sind. Das ist tatsächlich so, gilt jedoch bei nahezu allen Psychotherapien nach wie vor auch. Die klinische und neurobiologische Beforschung von EMDR hat auf der anderen Seite eine Vielzahl von Studien und interessanterweise einen Umkehreffekt hervorgebracht: Anhand der Versuche, eindeutig zu beweisen, wie und warum dieses (,exotische“) EMDR denn genau wirkt, ist breiteres Wissen über die Neurobiologie von Psychotherapie allgemein entstanden (Hofmann 2014).

So wurde z. B. der linke präfrontale Cortex, der am höchsten entwickelte Teil unseres Zentralnervensystems, als das Zentrum für höhere spezifische Erfahrungsintegration und Bewertung von Erfahrungen identifiziert, bei allem limitierten Wissen über die genauen Mechanismen. Nach einer EMDR Behandlung findet sich in bildgebenden Verfahren eine Aktivierung in genau diesen für Integration relevanten kortikalen Regionen (z. B. van der Kolk 2014, Jatzko und Ruf 2007, Pagani 2012). Realistischerweise muss natürlich gesagt werden, dass genau dieser Teil des Zentralnervensystems sicher einer der mit den komplexesten Aufgaben und Phänomenen ist und eine detaillierte Aussage über einzelne Aspekte noch nicht möglich ist.

Es gibt aufgrund der vielfältigen Forschung in verschiedene Richtungen selbstverständlich konkrete Hypothesen über die Wirkmechanismen: Es scheint eine Kombination folgender Aspekte zu sein, die die erstaunlichen Effekte hervorruft:

1. der spezifische therapeutische Halt im „Hier und Jetzt“ durch den/die TherapeutIn im Kontakt mit den körperlich-emotionell-kognitiv-sensorischen Belastungen des „Dort und Damals“, der sogenannten bifokalen Aufmerksamkeit (Dworkin 2005; Münker-Kramer 2015)

2. die genaue Fokussierung auf den Kern der Belastung auf allen Repräsentationsebenen im Vorfeld des freien Prozessierens in der oben kurz beschriebenen Phase 3, der Bewertungsphase (Shapiro 2012, 2013; Shapiro \& Silk-Forrest 2004)

3. die bilaterale Stimulation des Gehirns (Stickgold 2002) inklusive einer physiologischen Entlastung sowie
4. die Tatsache, dass das Arbeitsgedächtnis (working memory) durch die Außenorientierung in Form der (bilateralen) Stimulation von der inneren Belastung bzw. intrusiven Material abgelenkt ist (z. B. Theeuwes et al. 2009; Tsai und McNally 2013; Leer et al. 2014) und so die Belastungen reduziert wahrgenommen werden.

So zeigt sich klinisch die Wirkung von EMDR durch folgende Phänomene, die sonst nur durch langfristige intensive psychotherapeutische Arbeit auf allen Repräsentationsebenen erzeugt werden kann und dann auch oft nicht in diesem Ausmaß und Erleben:

- die Neu- oder Reattribuierung von Erinnerungen

- neue Etiketten für alte Erinnerungen/Erfahrungen

- neue Verbindungen zwischen Netzwerken

- neue Überschriften

- neue Einordnungen, Wiedereinordnungen

- Transformation passiert von Innen

- Anbinden an passendere Netzwerke passiert

- Konsolidierung

- neue Anpassungen

- Erinnerung verwandelt sich

- Verbindung zu Ressourcen

- physiologisch-emotionale Entlastung

Dieses autonom ablaufende Phänomen in der sogenannten Desensibilisierungs- und Reprozessierungsphase des EMDR (siehe oben, Phase 4) nennt man „R“ (für Re-Prozessieren). Bei Menschen mit überschaubaren Belastungsnetzwerken (sogenannte Typ I Traumatisierung, siehe auch Münker-Kramer 2009, 2011, 2015) bzw. bei spezifischen Indikationen ist es dies, was die Patienten benötigen zur deutlichen Entlastung, unterstützt eben durch bifokale Aufmerksamkeit und bilaterale Stimulation. Dies ist in solchen Fällen meist ausreichend.

Shapiro fiel diese inhaltliche autonome Transformation kurz nach der systematischen Erforschung der wahrgenommen Entlastungen bereits kurz nach der Beschreibung der Methode auf. Sie hatte zunächst angenommen, EMD (damals noch) sei eine reine Desensibilisierungsmethode, bei der in vitro in Kontakt mit Belastung gegangen werde, dann Desensibilisierung erfolge, die mit BLS (bilateraler Stimulation) unterstützt werde und dann rein die physiologischemotionelle Belastung sinke. Als sie feststellte, dass sich inhaltlich-qualitativ zusätzlich etwas änderte, erweiterte sie den Namen EMD zu EMDR (Shapiro 2013). Damit wollte sie genau diesem spezifischen inhaltlichen Phänomen in Phase 4 (Reprozessieren) Rechnung tragen: „Die Namensänderung von EMD zu EMDR im Jahre 1990 war ein Orientierungswechsel vom anfänglich verhaltenspsychologischen Ansatz einer einfachen Angst-Desensibilisierung hin zu einem umfassenderen Paradigma der Informationsverarbeitung." (Shapiro 2013, S. 42).

Interessant in diesem Kontext ist, dass immer wieder diskutiert wird, eben diesen Fokus im Begriff Re- 
prozessieren in der Bezeichnung der Methode zu verwenden, der sicher griffiger und klarer als EMDR wäre. Francine Shapiro hat sich dennoch entschieden, EMDR als Name zu belassen, zum einen aus historischen Gründen, zum anderen aus pragmatischen Gründen im Sinne der weltweiten Namensrechte und des Labels an sich. Sie merkt an, dass sie vor dem Hintergrund dessen, was sie heute weiß, unter Umständen „Reprocessing-Therapie“ wählen würde, wenn sie noch einmal einen Namen wählen müsste (Shapiro 2013).

\section{Gibt es eine Hintergrundtheorie zu EMDR?}

Wie bereits in den allgemeinen einleitenden Ausführungen erwähnt, soll EMDR physiologisch-emotionalkognitiv wahrgenommene Belastung, die spezifische Symptome wie z.B. in der klassischen PTBS (Posttraumatische Belastungsstörung) hervorbringt, in relativ kurzer Behandlungsdauer reduzieren. Dies erfolgt wie bei allen spezifischen Traumatherapiemethoden einerseits auf Basis der grundsätzlichen traumatherapeutischen Haltung (Reddemann 2005; Huber 2009; Hofmann 2014) und anderseits entlang den grundlegenden Phasen der Anamnese, Beziehungsaufbau, Stabilisierung, der Traumakonfrontation und Integration und der Neubewertung und Nacharbeit.

Shapiro entwickelte auf diesem Hintergrund sehr früh ihr bis heute gültiges Erklärungsmodell, das sie EMDR zugrunde legt: das Modell der Adaptiven Informationsverarbeitung (englisch: Adaptive Information Processing) - AIP.

Der oben beschriebene auffällige und erstaunliche inhaltliche und qualitative Veränderungsprozess im Erleben und Verhalten ist Anlass für die Arbeitshypothese dieses Modells. Es geht davon aus, dass in jedem Menschen ein inhärentes, immanentes, autonomes neurophysiologisches „System“ wirkt. Dieses strebt an, jegliche eintreffende Information im Sinne einer mental-emotional-physiologischen Homöostase einzuordnen, zu kategorisieren und zu integrieren und vermag dies auch für „normale Alltagsbelastungen" Hier sind die Hypothesen, die die Wirkweise von EMDR mit der sogenannten REM-Schlafphase in Zusammenhang bringen, interessant (Stickgold 2002). Auch im Schlaf machen wir die Erfahrung, dass sich Erinnerung konsolidiert und Erlebnisse sich quasi „über Nacht“ einordnen.

Wenn nun aber die Belastungen „katastrophales Ausmaß" mit Hilflosigkeitserfahrungen, Erschütterung des Selbst- und Weltverständnisses, Ausschaltung der Selbstwirksamkeit etc. erreichen (Fischer und Riedesser 2003), dann versagt dieses System bzw. ist überfordert. Die Erfahrungen werden nicht integriert und - umgangssprachlich gesprochen - bleiben im sogenannten impliziten (amydaloiden - „heißer Speicher") Gedächtnis stecken. Die Informationsverarbeitung und Speicherung ist vereitelt. Weiters ist die sprachlich-logisch-zeitliche Einordung - verbunden mit hohem Körperstress - blockiert, unter anderem durch die Blockade des Broca-Sprachareals. Aus dieser Kombination resultieren die Hauptsymptome wie Intrusionen und Hyperarousal (z.B. Huber 2009).

Gehalten durch die Präsenz des/der TherapeutIn im Hier und Jetzt scheint nun EMDR das Phänomen der Selbstheilung und Integration „nachzuvollziehen“ und so die Veränderungen und Einsichten doch noch zu ermöglichen (siehe Beschreibung oben). Somit können die Erlebnisse im sogenannten expliziten oder hippocampalen („kühler Speicher“) Gedächtnis eingeordnet werden. Genaueres, auch Hypothesen zu den neurobiologischen Hintergründen, findet sich bei Shapiro \& Silk Forrest 2004, Shapiro 2012, 2013); Hofmann (2014).

\section{EMDR praktisch - die Standards: Protokolle, Prozeduren, Techniken}

Wie bereits erwähnt, ist EMDR bereits sehr schnell (1995) standardisiert und der Versuch der Sicherstellung dieser Standards in Fachgesellschaften geregelt worden (Hofmann 2014; Münker-Kramer 2015). Der Hintergrund war, die Methode und damit die Betroffenen zu schützen: da man bei der beobachteten massiven und beeindruckenden und durchaus invasiven Wirkung (im positiven Sinne, wenn sachkundig appliziert) von „Nebenwirkungen“ bei unsachgemäßer Anwendung und mangelnder klinischer Erfahrung der BehandlerInnen ausging.

EMDR unterscheidet im Vorgehen zwischen Protokollen, Prozeduren und Techniken. Die Protokolle beschreiben die Logik der Behandlungsplanung, die Prozeduren das konkrete klinische Vorgehen im Einzelnen: „EMDR is an approach to psychotherapy that is comprised of principles, procedures and protocols and. It is not- as often depicted - a simple technique characterized primarily by the use of eye movements." (Luber 2009, Vorwort, keine Seitenzahl).

So geht man bei überschaubaren Belastungen nach folgendermaßen vor: die Belastungen der Vergangenheit, die man als „touchstone memories“ (Shapiro und Laliotis 2010) bezeichnet, werden zuerst bearbeitet und entlastet. Dann folgen die sogenannten Trigger in der Gegenwart, die sich aufgrund der alten Belastungen entwickelt haben. Zuletzt werden die Befürchtungen für die Zukunft bearbeitet, die durch die vorangegangene Schwächung entstanden sind. So kann z. B. ein schwerer Autounfall in der Vergangenheit zu gefährlicher und belastender Unsicherheit bei ähnlichen Situationen im Straßenverkehr in der Gegenwart führen und hier Probleme machen. Er kann auch hervorrufen, dass die Perspektive entsteht, gar nicht mehr Autofahren zu wollen oder zu können und die Lebensqualität oder Beweglichkeit massiv leidet.

In dieser Reihenfolge werden im sogenannten Standardprotokoll die Beeinträchtigungen mit EMDR Sitzungen bearbeitet (Münker-Kramer 2014, 2015). 
Bei komplexeren, weniger monokausalen Belastungsnetzwerken wird umgekehrt vorgegangen. Hier wird außerdem vor der Bearbeitung der Erinnerungen zusätzlich gezielt Kontakt mit hilfreichen realen ressourcenreichen „Gegensituationen“ aufgenommen und mittels EMDR Techniken „verankert“ (z.B. Rost 2013). Dann beginnt man mit den angenommenen Einschränkungen in der Zukunft, die in den alten Belastungen ihren Ursprung haben könnten. In der Folge behandelt man die Auslöser in der Gegenwart und wendet sich dann zunächst dem sekundären Material der Vergangenheit zu. Zuletzt, und sehr dosiert, werden Primärbelastungen der Vergangenheit avisiert. So könnte z. B. eine von Gewalt geprägte Kindheit im Hintergrund sein und eine Patientin könnte nach einer Trennung in der Gegenwart Herausforderungen zu bewältigen haben. Diese sollten in dem Fall zuerst bearbeitet werden, um im Hier und Jetzt grundsätzliche Stabilität $\mathrm{zu}$ erzeugen und Selbstwirksamkeit erlebbar zu machen. Beispiele wären Angst vor Bewerbungsgesprächen mit potentiell autoritär wirkenden Gesprächspartnern nach Arbeitsplatzverlust oder Behördenkontakte sein.

Für nicht spezifisch klassischen traumatischen Erlebnissen zuzuordnenden Beschwerden werden in adaptierten „Protokollen“ weitere Behandlungsplanungen vorgeschlagen, wie z. B. bei Zwangssymptomatik, bei spezifischen Phobien, Sucht, bei anderen Zielgruppen wie Kindern, Akuttraumatisierten u.

Neben diesen differenzierten Überlegungen zur optimalen Behandlungsplanung (Protokoll) bedient sich das EMDR konkreter sehr standardisierter Ablaufschemata in der konkreten Sitzung (Prozeduren). Hier wird, wie oben erwähnt, die Belastung bewertet, durchgearbeitet (desensibilisiert, reprozessiert), unter Integration der neuen Erkenntnisse und modifizierten Erlebens und Bewertens verankert, der Körpertest durchgeführt und die Sitzung nachbesprochen (Phase 3-7). Auch hier gibt es Modifikationen bei spezifischen Beschwerden wie Zwängen, Alpträumen, Sucht und auch wieder bei speziellen Zielgruppen wie oben.

Unterstützt wird die Navigation durch die Protokolle und Prozeduren durch Einzeltechniken wie besonderen Ressourcentechniken, die mittels bilateraler Stimulation in ihrer Wirksamkeit verstärkt werden - wie die sogenannte Absorptionstechnik oder Kurzkonfrontationstechniken wie CIPOS (Constant Installation of Present Orientation and Safety), die flexibel im Kontext von klassischem EMDR zur Stabilisierung und Vorbereitung auf Konfrontation oder auch per se eingesetzt werden können (vgl. im Detail auch Rost und Münker-Kramer 2016).

\section{Struktur und Kreativität im EMDR - ein Widerspruch?}

All diese Bausteine werden nach klar vorgegebenen, gut durchdachten und in Studien evaluierten Regeln appliziert und entfalten so ihre Wirkung. Diese starke Übereinstimmung zwischen ursprünglicher Theorie und konkreter Praxis und Ausbildung kann vordergründig als sehr rigide wahrgenommen werden. Auf der anderen Seite aber enthält der EMDR Prozess in der sehr autonom ablaufenden Phase des Reprozessierens sehr viel immanente Kreativität. Dies ist eine nahezu widersprüchliche Mischung in sich.

Es scheint so, als müsse der Veränderungsprozess im EMDR sinnvollerweise und sorgfältig eingebettet sein in einen klaren Rahmen, um gezielt heilsam $\mathrm{zu}$ sein.

Rost führt dies folgendermaßen aus: „EMDR an sich ist extrem kreativ ....wir erleben eine beschleunigte Verarbeitung, die ausgesprochen kreativ sein kann, mit neuen Erkenntnissen und Verknüpfungen ..., mit imaginativen Lösungsmöglichkeiten und/oder Veränderungen .... Keine EMDR Sitzung verläuft wie die andere, selbst bei ein und derselben Patientin nicht. Wir steuern nicht, wir schaffen nur die passenden Rahmenbedingungen - und zwar mit unserer Struktur, in der der Prozess sich dann (von uns) möglichst ungestört entwickeln kann. Im Prozess der Verarbeitung erleben wir beim EMDR also eine enorme Kreativität.“ (Rost 2016, S. 11).

Zitate von PatientInnen dazu finden sich zahlreich (hier einige von der Autorin von den PatientInnen zusammengetragene Beispiele):

- „man sieht das Hirn arbeiten“

- „eigentlich müsste man eine Kino-Eintrittskarte zahlen"

- „das Kopfkino“, „der Comic“

- „bürokratisches Voodoo“

Zur Frage „Wie passen Theorie und klinische Praxis zusammen“ - eine Leitfrage in dieser Schwerpunktnummer der Zeitschrift - finden sich bei EMDR nun durchaus spezielle Bedingungen. Wie aus den vorherigen Ausführungen hervorgeht, scheint es, dass das Vorgehen in den Kernphasen nach den sehr klaren Vorgaben weniger eine ideologische Haltung als ein optimales Service für die PatientInnen ist. Es ist auch nicht $\mathrm{zu}$ verwechseln mit „Schema F“, da es keine Simplifizierung ist. Es bedarf im Gegenteil einfach an bestimmten Punkten spezieller klinischer Präzision und genauer Kenntnis dessen, was man denn da nun warum und wie macht und warum man innerhalb der Kernphasen den PatientInnen keinen Gefallen mit ungezielter und eher subjektiv motivierter „Kreativität“ tut.

Problematisch wird es auch, wenn jemand EMDR nicht eingebettet ins AIP-Modell sieht (i.S. von Bearbeitung dysfunktionaler und pathogener Erinnerungen nach Plan), sondern als „Technik an sich“, die zudem noch spektakulär wirkt und ggf. das eigene Image aufpoliert.

Dies alles ist nicht nur nicht zielführend, es sind oft Modifikationen, die weder not-wendig im wahrsten Sinne des Wortes noch klinisch begründbar sind. 
Ein Steuern innerhalb des gut erarbeiteten und vorgegebenen Rahmens auf Basis klinischer Erfahrung ist das beste Service für die PatientInnen, die sich EMDR TherapeutInnen in der Hoffnung auf Erleichterung und seriöse Behandlung anvertrauen.

Platz und Gelegenheit für sinnvolle und heilsame Kreativität ist zwischen den einzelnen Elementen von EMDR, in der Behandlungsplanung (Betonung auf Planung), in der Stabilisierung, in der Gesamttherapie. Auch von einer/m ChirurgIn erwartet man Präzision im Sinne der Richtlinien. Modifikationen und Diskussionen in der OP-Vorbereitung und in der Nachbehandlung sind ggf. sinnvoll, aber nicht mitten in der OP. EMDR ist mit präzisen Operationen am definierten, fokussierten, sterilisierten und vorbereiteten OP Feld vergleichbar.

Jenny Ann Rydberg, eine französische EMDR Supervisorin, fasst dies in engagierten Worten rund um dieses Thema folgendermaßen zusammen: „Do we want to suggest that each diagnosis, each clinical population, each particular context or circumstance requires its own specific protocol or that the standard protocol may be used for most if not all psychological and psychosomatic symptoms - albeit with a set of „standard variations“, which are part of the basic principles, protocols, and procedures of EMDR?“ (Rydberg 2016, 2017). Dies wäre der Aufruf, nicht jede kleine klinisch notwendige Modifikation ein neues Protokoll zu nennen, sondern theoretisch und klinisch zu wissen, was man gerade tut innerhalb des beschriebenen Vorgehens.

Zusammenfassend dazu lässt sich also festhalten, dass eine Anwendung auf folgenden Säulen basieren sollte:

- Grundverständnis aus dem AIP Modell heraus, Integration von EMDR in die Gesamttherapie

- traumatherapeutische Grundhaltung nach sorgfältiger Anamnese

- Phasenbeachtung

- Behandlungsplanung, Indikationsstellung, sorgfältiger Targetauswahl in Phase 3

- Fokussierung und Verständnis, um was es geht

- profundes klinisches Verständnis und Haltefunktion im „Hier und Jetzt“

- Modifikationen und Techniken nur da, wo „notwendig“, Rückkehr so schnell wie möglich, kurz „so viel Kreativität wie nötig, soviel Standard wie möglich“

\section{Ausblick}

EMDR wurde lange vordergründig als Methode zur Bearbeitung traumatischer Erfahrungen verschiedener Art, aber primär der PTBS verstanden, da sie sich historisch entlang dieser Störungen entwickelt und ihre Wirksamkeit bewiesen hatte (s. unten). Nach vielen Jahren der unermüdlichen Arbeit an Beforschung, Anerkennung, Manualisierung (Luber 2009,
2010, 2014) und Standardisierung von EMDR in diesem spezifischen Bereich erfolgte in den letzten fünf bis sieben Jahren ein weiterer Paradigmenwechsel: die Öffnung der empfohlenen Anwendungsbereiche auch über diesen klassischen Bereich hinaus und in letzter Zeit wird sogar die Überlegung laut, EMDR als eigene Psychotherapiemethode zur Bearbeitung jeglicher auf pathogenen Erinnerungen basierender Störungen zu verstehen. Dies passt sicher zu den aktuellsten diesbezüglichen neurobiologischen Forschungen über die Repräsentanz psychischer Störungen im ZNS.

\section{Zusammenfassung und Schlussfolgerungen}

Eine verantwortungsvolle Anwendung von EMDR im Sinne der Methode, aber vor allem im Sinne der PatientInnen ist eine wesentliche Grundaussage des Artikels und begründet sowohl die Grundstruktur als auch die sorgfältige Entwicklung und differenzierte Vermittlung von Modifikationen.

Dies hat Implikationen für Lehre, Forschung, Publikationen, nationale und internationale Fachgesellschaften und klinische Praxis und mag mitunter spröde und unflexibel wirken, sollte aber letztlich dazu dienen, den Benefit, den die Methode für die Betroffenen bietet, in höchstmöglicher Qualität zu erhalten.

\section{Internetlinks (Ausbildungsinstitute, Fachgesell-} schaften, Information)

- www.emdr-institut.at, www.emdr.de, www.emdr. com, www.emdria.org, www.emdr-europe.org, www. emdr-netzwerk.at

- https://www.youtube.com/watch?v=aTyZWRCUhR Q\&feature=youtu.be - Allgemeine Einführung

- https://www.youtube.com/watch?v=KFvxmCi5LM Q\&feature=youtu.be - Depressionen, Phobien und gute EMDR Therapeuten

- https://www.youtube.com/watch?v=vStJ5indPFw\& feature=youtu.be - EMDR International

Interessenkonflikt E. Münker-Kramer gibt an, dass kein Interessenkonflikt besteht.

Open Access Dieser Artikel wird unter der Creative Commons Namensnennung 4.0 International Lizenz (http:// creativecommons.org/licenses/by/4.0/deed.de) veröffentlicht, welche die Nutzung, Vervielfältigung, Bearbeitung, Verbreitung und Wiedergabe in jeglichem Medium und Format erlaubt, sofern Sie den/die ursprünglichen Autor(en) und die Quelle ordnungsgemäß nennen, einen Link zur Creative Commons Lizenz beifügen und angeben, ob Änderungen vorgenommen wurden.

\section{Literatur}

Bisson, J. (2013). Psychological therapies for chronic PTSD in adults (review). The Cochrane review. Hoboken: John Wiley\&Son. 
Dworkin, M. (2005). EMDR and the relational imperative. The therapeutic relationship in EMDR treatment. New York: Routledge.

Fischer, G., \&Riedesser, P. (2003).Lehrbuch der Psychotraumatologie. Stuttgart: Reinhardt

G-BA Deutschland. https://www.g-ba.de/institution/presse/ pressemitteilungen/557/.Zugegriffen: 15.Jan. 2017.

Hofmann, A. (Hrsg.). (2014). EMDR - Praxishandbuch zur Behandlung traumatisierter Menschen (5. Aufl.). Stuttgart: Thieme.

Huber, M. (2009). Trauma und die Folgen. Paderborn: Junfermann.

Jatzko, A., \& Ruf, M. (2007). Durch EMDR normalisierte funktionelle Verarbeitungsprozesse bei PTBS, eine fMRT Pilotstudie. Poster. DeGPT Konferenz2007.

van der Kolk, B. (2014). The Body keeps the Score. New York: Penguin.

Leer, A., Engelhard, I. M., \& van den Hout, M.A. (2014). How eye movements in EMDR work: changes in memory vividness and emotionality. Journal of Behavior Therapy and Experimental Psychiatrydoi:10.1016/j.jbtep.2014.04.004.

Luber, M. (2009). Scripted protocols. Basics and Special Situations. New York: Springer

Luber, M. (2010). Scripted protocols. Special Populations. New York: Springer

Luber, M. (2014). Scripted protocols. EMDR Early Mental Health Interventions. NewYork: Springer

Münker-Kramer, E. (2009). Eustress - Distress - Extremstress und was dann? Folgestörungen und Behandlungsansätze. Psychologie in Österreich, 29(1), 54-62.

Münker-Kramer, E. (2011). EMDR und VT in der Behandlung von Traumafolgestörungen - Charakteristika und Kombinations-möglichkeiten. Zwischen Alltag und ICD-10: Trauma-Krise-Belastung-Extremstress, Journal für Psychologie (3), open access Zeitschrift. http://www.journalfuer-psychologie.de.Zugegriffen:22.3.2017.

Münker-Kramer, E. (2014). EMDR in der Traumatherapie, Psychopraxis, Neuropraxis, 1-2014 (S. 13-16). Berlin: Springer. doi:10.1007/s00739-013-0136-3. letzter Zugriff $17.2 .2017,10.14 \mathrm{~h}$

Münker-Kramer, E. (2015). Traumazentrierte Psychotherapie mit EMDR. München: Ernst Reinhardt.

NICE Report (2005). National clinical practice guideline no. 26: post traumatic stress disorder. The management of PTSD in adults and children in primary and secondary care. Gaskell \& British Psychological Society. www.nice. org.uk

Pagani, M., Di Lorenzo, G., Verardo, R., Nicolais, G., Monaco, L., Lauretti, G., Russo, R., Niolu, C., Ammaniti, M., Fernandez, I., \& Siracusano, A. (2012). Neurobiological Correclates of EMDR Monitoring - An EEG Study. PloS one, 9(7),e45753 (open access).

Power, K., McGoldrick, T., Brown, K., Buchanan, R., Sharp, D., Swanson, V., \& Karatzias, A. (2002). A controlled compari- son of EMDR versus exposure plus cognitive restructuring versus waiting list in the treatment of PTBS. Clinical Psychology and Psychotherapy, 9, 299-318.

Reddemann, L. (2005). Psychodynamisch-Imaginative Traumatherapie. PITT - Das Manual. Stuttgart: Pfeiffer bei Klett-Cotta.

Rost, C. (Hrsg.). (2013). Ressourcenarbeit mit EMDR. Paderborn: Junfermann.

Rost, C. \& Münker-Kramer, E. (Hrsg.). (2016). Bewährte Techniken im Überblick. In Rost, C., EMDR zwischen Struktur und Kreativität(S. 129-139). Paderborn: Junfermann.

Rydberg, J.A. (2016). Navigating the EMDR special protocols. Den Haag: EMDR Europe Congresses Edinburgh. 1. Auflage: 2014

Rydberg, J.A. (2017). Protocoles EMDR spécialisés. In C. Tarquinio, M. J. Brennstuhl, H. Delluci, M. Iracane, J. A. Rydberg, M. Silvestre \& E. Zimmermann (Hrsg.), Pratique de la Psychothérapie EMDR(S. 117-127). Malakoff: Dunod.

Schulz, S., Dahm, A., Herrmann-Frank, A., Martinsohn-Schittkowsky, W., Nocon, M., \& Sühlfleisch-Thurau, U. (2015). Eye Movement Desensitization and Reprocessing (EMDR): Eine Methode wird anerkannt. Ärzteblatt online, 13(01), 34-36.

Shapiro, F., \& Silk Forrest, M. (2004). The breakthrough „Eye Movement" therapy for overcoming anxiety, stress and trauma. NewYork: Basis Books, Perseus Books Group.

Shapiro, F., \& Laliotis, D. (2010). EMDR and the adaptive information processing model: integrative treatment and case conceptualization. J. Clin. Social Work, 39, 191-200. doi:10.1007/s10615-010-0300-7.

Shapiro, F. (2012). Les Thérapies EMDR: une vue d'ensemble de la recherce actuelle et un apercu de la recherche future. Spezialausgabe Revue Européenne de psychologie appliqueé, 62, 193-195.

Shapiro, F. (2013). EMDR Grundlagen und Praxis. Paderborn: Junfermann.

Stickgold, R. (2002). EMDR: A putative neurobiological mechanism of action. Journal of Clinical Psychology, 58(1), $61-75$.

Theeuwes, J., Belopolsky, A., \& Olivers, C. (2009). Interactions between working memory, attention and eye movements. Acta Psychologica, 132, 106-114.

Tsai, C., \& McNally, R. J. (2013). Effects of emotionally valented working memory taxation on negative memories. Jour nal of behavior therapy and experimental psychiatry, 45, $15-19$.

Van Etten, M., \& Taylor, S. (1998). Comparative efficacy of treatments for post traumatic stress disorder: a meta analysis. Clinical Psychology and Psychotherapy, 5, 126-114.

WHO (2013). WHO mhGAP Guidelines. Problems and disorders related to Stress. Geneva, WHO. http://www.who. int/mediacentre/news/releases/2013/trauma_mental health_20130806/en/.Zugegriffen: 14.4.2017. 